\title{
Practical Utilization of Uranium-Containing Particulate Test Samples for SEM/EDS and SIMS Automated Particle Analysis Method Validation
}

\author{
Matthew S. Wellons ${ }^{1}$, Michael A. DeVore $\mathrm{II}^{1}$, Robert M. Rogers ${ }^{1}$, Joshua T. Hewitt ${ }^{1}$, Todd L. \\ Williamson ${ }^{2}$, Travis J. Tenner ${ }^{2}$ and Taghi Darroudi ${ }^{3}$ \\ ${ }^{1 .}$ National Security Directorate, Savannah River National Laboratory, Aiken SC, USA. \\ 2. Chemistry Division, Los Alamos National Laboratory, Los Alamos NM, USA. \\ 3. Clemson Microscopy Center, Clemson University, Anderson SC, USA.
}

The detection and characterization of uranium particulates from swipe samples collected by nuclear facilities safeguards inspectors often relies on automated search algorithms and instrument software for analysis via scanning electron microscopy (SEM) and/or secondary ionization mass spectrometry (SIMS). Because safeguards samples are inherently cluttered with background environmental material automated particle measurement (APM) methods with correctly tuned instrument parameters are required for robust identification of uranium containing material. Unfortunately, no standard or reference specimens are commercially available for uranium APM method validation or instrument proficiency testing. To meet such challenges the safeguards community has generated and characterized select test specimens over the past decade, but these efforts have been hindered by limited production and challenging characterization [1-3].

The dearth of reference materials has led Laboratories to generate in-house APM validation test samples. To this end, SRNL has developed a method that allows for rapid generation of uranyl oxalate particulates with uniform composition and size. Our technique utilizes conventional aerosol generation and in-house collection/deposition tools to simultaneously create multiple substrates with microgram to nanogram total uranium mass loadings [4]. Characterization of individual particles by micro-Raman spectroscopy and TEM of lamella (post FIB processing) have verified the uranyl oxalate species as anhydrous by both vibrational modes and lattice spacing, respectively, as shown in Figure 1. Sample generation parameters may be tuned to produce multiple specimens simultaneously with a desired mass/particle loading. A prototypical specimen contains approximately $\sim 1000$ spherical uranium particulates $\left(\sim 5\right.$ particles $\left./ \mathrm{mm}^{2}\right)$, a monomodal particle size distribution with average diameter of 1.8 microns ( $\sigma=0.4$ microns), and approximately $10^{-11}$ grams of uranium per particle. The sparse particulate density and low $U$ mass make these samples ideal for method validation for trace detection/characterization of actinides by automated SEM/EDS or SIMS.

Coupling the known uranyl oxalate test sample with carbon stub blanks in an end-to-end serial analysis, similar to standard GSR procedures, allows one to monitor instrument stability and operator efficacy via metrics such as particle count, position, and size distributions. Panel 1 of Figure 2 details the area and number of particles found in the standard analyzed at the start and end of two analytical queues. A shift in overall particle count and/or particle area distribution typically coincides with stage, detector electronics, or filament saturation drift. In practice, these failures often manifest as a decrease in per particle average area and/or reduction in particle count. Uranyl oxalate test specimens are also used to characterize SIMS APM method efficacy. Panel 2 of Figure 2 shows a 235/238 uranium isotopic ratio verse intensity plot for $>1000$ particulates as a means to probe measurement variance at reduced ion intensities. Other applications for uranyl oxalate test samples include correlation microscopy exercises between SEM and SIMS to characterization the effects of particle size on SIMS ionization yield, inter- 
instrument positional accuracy, and as bench mark for particulate chemical identification. SRNL has found that consistent use of these validation samples increase our analysis confidence levels and facilitates interlaboratory assessments of both SEM/EDS and SIMS APM methods.

\section{References:}

[1] M. Kraiem et al, Anal. Chim. Acta 748 (2012), p. 37.

[2] D. Simons, J. Trace Microprobe Tech. 4 (1986), p. 185.

[3] N. Sharp, J. Fassett and D. Simons, J. Vac. Sci. Technol. B. 34 (2016), p. 03H115/1.

[4] N. Bridges et al in "2013 Savannah River National Laboratory LDRD Annual Report \#SRNL-STI2014-00096", (SRNL, Aiken SC) p. 6.
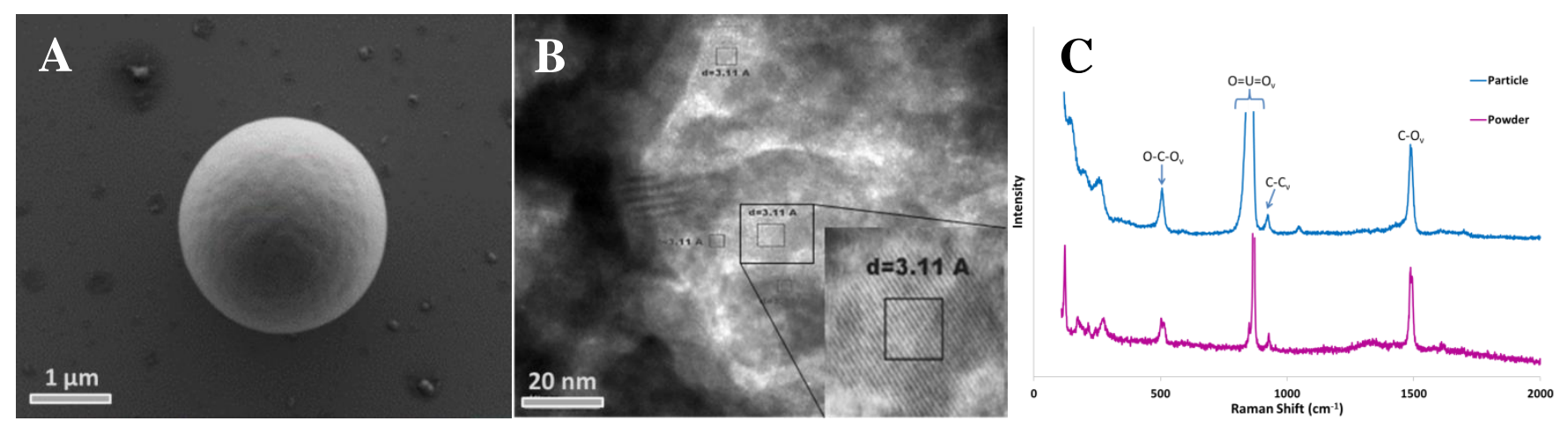

Figure 1. (A) Typical micron-sized uranyl oxalate particulate located on a validation specimen; (B) Post FIB processed uranyl oxalate particle lamella, characterized by TEM with lattice spacing consistent with anhydrous uranyl oxalate; (C) Raman spectra with consistent vibrational bands between anhydrous uranyl oxalate commercial powder and a representative validation specimen particulate.
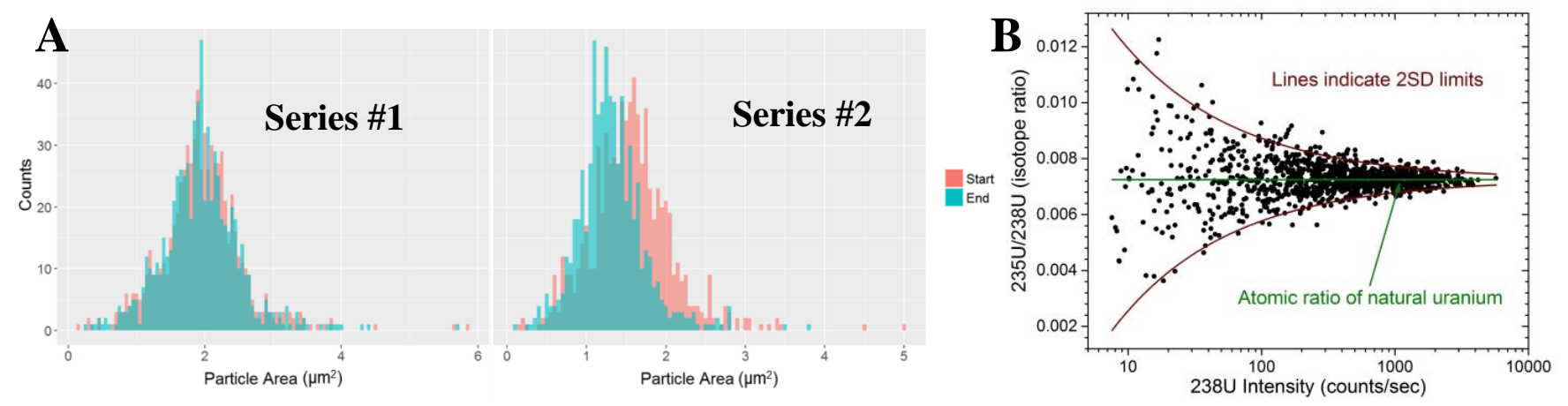

Figure 2. (A) Histograms of the same validation sample analyzed in two different sample analysis queues on a FEI Aspex Explorer SEM. Series 1 shows consistent particle area and number found for the analysis conducted at the beginning and end of the sample queue. Series 2 shows roughly equivalent total number of particles identified in the start and end of the sample queue but a strong shift in particle area. This suggests stage drift between the end-to-end APM analyses; (B) Plot of the 235/238 U isotope ratio per 238 U counts per sec collected on a Cameca IMS 1280 LG-SIMS (n=1134). 